\title{
Letting the right one in: evaluating the generalisability of clinical trials
}

\author{
Rahul Shrimanker ${ }^{1}$, Richard Beasley (i) $^{2}$ and Ciléin Kearns ${ }^{2}$
}

Affiliations: ${ }^{1}$ Respiratory Medicine Unit and Oxford Respiratory NIHR Biomedical Research Centre, Nuffield Dept of Medicine, University of Oxford, Oxford, UK. ${ }^{2}$ Medical Research Institute of New Zealand, Wellington, New Zealand.

Correspondence: Rahul Shrimanker, Respiratory Medicine Unit and NIHR Respiratory BRC, Nuffield Department of Medicine, NDM Research Building, Old Road Campus, University of Oxford, Oxford, OX3 7FZ, UK. E-mail: rahul.shrimankerandm.ox.ac.uk

@ERSpublications

Clinical trials can be poorly generalisable to a patient population due to strict, label-based diagnostic criteria. Selection using the trait the treatment is targeting may produce results more applicable to the disease population. http://ow.ly/p30b30mKrC5

Cite this article as: Shrimanker R, Beasley R, Kearns C. Letting the right one in: evaluating the generalisability of clinical trials. Eur Respir J 2018; 52: 1802218 [https://doi.org/10.1183/13993003.022182018].

Well-designed randomised controlled trials (RCTs) are the cornerstone of clinical research; they are the "gold standard" in comparing the efficacy and safety of two or more treatments in the fairest way. RCTs are essential in gaining regulatory approvals for new drugs; the US Food and Drug Administration (FDA) and the European Medicines Agency (EMA) used supporting RCTs in 90\% of their approvals from 1999 to $2014[1,2]$. The results from RCTs also provide the basis for recommendations made by guidelines, which inform evidence-based clinical practice.

A number of factors need to be considered when assessing the external validity, or generalisability, of RCTs, i.e. the degree to which the results apply to patients treated in routine clinical practice. These include, but are not limited to, the study population, outcome measures, trial duration, and the trial setting [3]. The inevitable conflict that arises with the requirement to achieve strong internal validity in the design of RCTs, in particular to meet stringent study inclusion and exclusion criteria to reduce bias, has meant that the generalisability of study findings to typical patient populations with the disease of interest can be poor [4].

The extent to which this limitation may apply to asthma has been shown by analyses which identified that the vast majority of adults with current asthma on treatment, in either primary or secondary care, would not have been eligible for inclusion in the major RCTs on which the international asthma guidelines are based $[5,6]$. Highly selected populations were studied in these RCTs to achieve strong internal validity by confirming diagnostic labels, for example, excluding smokers to avoid including patients with chronic obstructive pulmonary disease (COPD), but thereby excluding around a third of patients with asthma, an important high-risk subgroup with a differential response to treatments such as inhaled steroids [7]. Similarly, the requirement to only include patients with substantive bronchodilator reversibility excludes most patients with asthma, as this characteristic is only present in a minority of patients with asthma on the basis of a single test [8]. The clinical implication is that there is not an adequate evidence base for the management of the majority of adults with asthma, with the inevitable risk of inappropriate overtreatment and/or undertreatment. 
This issue has come into sharp focus with increased understanding of the heterogeneity in asthma, with recognition of clinically relevant phenotypes, such as type- 2 high, eosinophilic patients. This has led to treatment options targeted at specific type-2 phenotypes [9], based on phenotype-specific clinical trials $[10,11]$. Importantly, there is now a portfolio of monoclonal antibody therapies directed against specific type-2 targets, in which efficacy has been demonstrated in RCTs. Not least due to the high expense of these medications, it is necessary to scrutinise the external validity of the trials, to determine which specific populations should be preferentially considered for funding and prescription of these novel agents.

This has been addressed in the current issue of the European Respiratory Journal, in which Brown et al. [12] assess the eligibility of patients in their tertiary care severe asthma cohort, the Wessex Severe Asthma Cohort (WSAC), to enter RCTs of novel treatments for asthma. Their patients underwent detailed clinical, physiological and biological characterisation, and had confirmed severe asthma as per the American Thoracic Society/European Respiratory Society 2014 definition [13]. The authors report that $9.8 \%$ of their 342 patients with severe asthma would have been eligible for trials of biological treatments for severe asthma. A finding that a minority of patients were eligible may not have been surprising, as most of these trials targeting a specific part of the type-2 pathway required the presence of type-2 biology, a characteristic not required to be included in the WSAC cohort. Indeed, previous studies of mepolizumab have shown that applying type-2 targeted treatments to an unselected severe asthma cohort can mask the beneficial effects of the drug [14], which are clearly seen when tested in the right population [10]. However, the figure of around only one in 10 patients being eligible is extremely low and suggests poor generalisability of the trial's findings to a population with refractory severe asthma. Importantly, the authors report that the majority of patients who met the study's biomarker requirements were excluded due to criteria measuring airflow obstruction and/or variability. For example, only $21.1 \%$ of patients with severe eosinophilic asthma would have been eligible for inclusion in the phase III licensing trials of interleukin (IL)-5/5R targeted therapies. Thus, the vast majority of patients were excluded from trial participation by criteria designed to reconfirm diagnostic labels, rather than by biomarker criteria that identify the type- 2 characteristics, and importantly predict responsiveness to treatment.

The funding and clinical availability of these new biological drugs is another consideration, and it is of interest that $50 \%$ of the WSAC cohort who are eligible for the anti-IL-5 monoclonal antibodies mepolizumab and reslizumab according to the UK National Institute for Health Care and Excellence (NICE) criteria would not have been eligible for the clinical trials which formed part of the NICE technology appraisals. NICE and other commissioning bodies have to apply their own methods to generalise the findings from RCTs conducted in narrow trial populations to a wider disease population.

The impact of patient selection criteria was evident from the 37 studies evaluated by BRown et al. [12], all of which evaluated drugs in severe asthma, in which 12 different criteria were used to define airflow obstruction. Some studies allowed one of a number of criteria to be used to demonstrate this and/or bronchodilator reversibility, but this only modestly increased trial eligibility. Differing criteria for defining a characteristic makes it increasingly difficult to generalise and apply RCT findings not only to a real-world patient, but also to other clinical trials.

Another question that needs to be asked is "what is the rationale for, and implications of the use of specific selection criteria?" In the case of type- 2 targeted biological treatments, the desired effect is a reduction in airway inflammation and a subsequent reduction in exacerbation risk. Forced expiratory volume in $1 \mathrm{~s}$ (FEV1), $\mathrm{FEV} 1 /$ forced vital capacity ratio or bronchodilator reversibility have not been shown to predict a response to type- 2 targeted treatments, and in some cases are negatively associated with response to treatment [15]. By contrast, markers of type-2 biology such as the peripheral blood eosinophil count or the exhaled nitric oxide fraction have been shown to predict response [16]. Diagnostic labels may also unnecessarily restrict the external validity of monoclonal antibody therapies for type- 2 high inflammation. For example, mepolizumab has been shown to reduce exacerbations in asthma [10] and, to a lesser extent, in COPD [17] in patients with raised blood eosinophil counts regardless of the diagnostic label or lung function.

More broadly, the identification of individual causal mechanisms within a patient that have specific treatments is the basis of the "treatable traits" approach to the management of airways disease [18]. If the requirement for diagnostic labels which do not influence the treatment efficacy was removed, the focus could instead be on the biological trait of interest (figure 1).

Agusti et al. [18] suggest a number of treatable traits in airways disease, including pulmonary traits such as airflow limitation and eosinophilic airway inflammation, extrapulmonary traits such as chronic rhinosinusitis and vocal cord dysfunction, and behavioural/lifestyle risk factors such as adherence, inhaler technique and exposure to sensitising agents. The challenge now is to determine not only the clinical utility of recognising and treating specific treatable traits, but also a practical system whereby this approach 


\section{"Diagnostic labelling" approach}

Cohort selected by diagnostic label criteria, e.g. severe asthma, nonsmoker, with reversible bronchoconstriction.

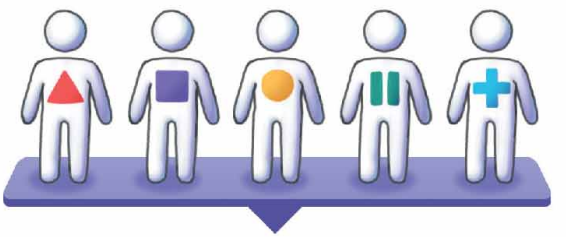

Resulting cohort has a mix of disease process phenotypes, and may be limited more than necessary.
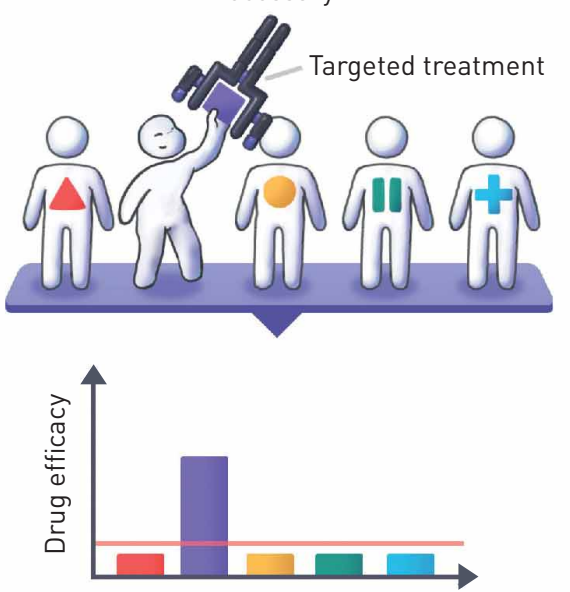

Outcome: Cohort may include participants whose disease traits are not targeted by treatment, and exclude those with treatable traits who do not fit diagnostic labels. External validity of results is compromised.

\section{"Treatable trait" approach}

Cohort selected by "treatable trait" biomarker which predicts response to targeted treatment, e.g. type-2 inflammation driven asthma.

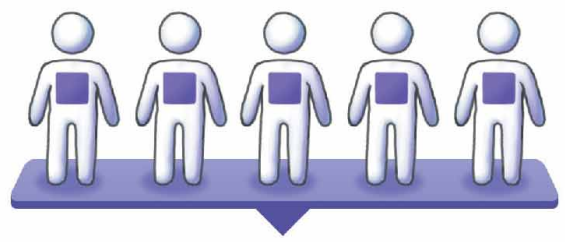

Resulting cohort is representative of the disease process the treatment targets.
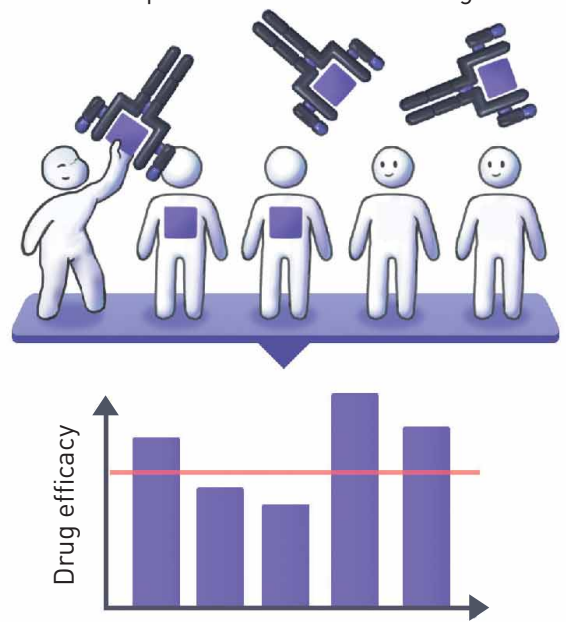

Outcome: Cohort selection by "treatable trait" biomarker of disease process results in wider pool of eligible, representative participants. External validity of results is improved for targeted therapy efficacy.

FIGURE 1 External validity of randomised controlled trial results for targeted therapy is limited by a "diagnostic labelling" approach to participant eligibility criteria.

might be employed in clinical practice, both in primary and secondary care [19]. It will also be necessary to determine, for each trait, their prevalence in specific populations, their ease of diagnosis and the investigations required, the burden of disease, and the efficacy, safety and cost of treatment. These issues have been considered in a recent review of the Australasian Severe Asthma Web-based Database, in which MCDonald et al. [20] explored the prevalence of 24 traits in two populations, and their impact on exacerbation risk. This approach allows the traits to be triaged by their prevalence, relative risks, and the impact of treatments on these risks [21].

The identification of type-2 phenotypes for which specific treatments are targeted, regardless of diagnostic labels, is arguably the best example of the utility and importance of the treatable traits approach in airways disease [22]. It will be important to learn from the experience obtained with the research programmes of the type-2 biologic agents in the assessment of other potential treatable traits in airways disease. This includes the internal and external validity of the RCTs on which their use in clinical practice, and funding, will be based.

Conflict of interest: R. Shrimanker has nothing to disclose. R. Beasley reports grants from Health Research Council of New Zealand and Genentech, grants and personal fees from Astra Zeneca and GlaxoSmithKilne, outside the submitted work. C. Kearns has nothing to disclose. 


\section{References}

1 Hatswell AJ, Baio G, Berlin JA, et al. Regulatory approval of pharmaceuticals without a randomised controlled study: analysis of EMA and FDA approvals 1999-2014. BMJ Open 2016; 6: e011666.

2 Downing NS, Aminawung JA, Shah ND, et al. Clinical trial evidence supporting FDA approval of novel therapeutic agents, 2005-2012. JAMA 2014; 311: 368-377.

3 Rothwell PM. External validity of randomised controlled trials: "to whom do the results of this trial apply?" Lancet 2005; 365: 82-93.

4 Halpin DM, Kerkhof M, Soriano JB, et al. Eligibility of real-life patients with COPD for inclusion in trials of inhaled long-acting bronchodilator therapy. Respir Res 2016; 17: 120.

5 Travers J, Marsh S, Williams M, et al. External validity of randomised controlled trials in asthma: to whom do the results of the trials apply? Thorax 2007; 62: 219-223.

6 Herland K, Akselsen JP, Skjonsberg OH, et al. How representative are clinical study patients with asthma or COPD for a larger "real life" population of patients with obstructive lung disease? Respir Med 2005; 99: 11-19.

7 Chalmers GW, Macleod KJ, Little SA, et al. Influence of cigarette smoking on inhaled corticosteroid treatment in mild asthma. Thorax 2002; 57: 226-230.

8 Fingleton J, Weatherall M, Beasley R. Bronchodilator responsiveness: interpret with caution. Thorax 2012; 67: 667-668.

9 Pavord ID, Hilvering B, Shrimanker R. Emerging biologics in severe asthma. Immunol Allergy Clin North Am 2016; 36: 609-623.

10 Pavord ID, Korn S, Howarth P, et al. Mepolizumab for severe eosinophilic asthma (DREAM): a multicentre, double-blind, placebo-controlled trial. Lancet 2012; 380: 651-659.

11 FitzGerald JM, Bleecker ER, Nair P, et al. Benralizumab, an anti-interleukin-5 receptor alpha monoclonal antibody, as add-on treatment for patients with severe, uncontrolled, eosinophilic asthma (CALIMA): a randomised, double-blind, placebo-controlled phase 3 trial. Lancet 2016; 388: 2128-2141.

12 Brown T, Jones T, Gove K, et al. Randomised controlled trials in severe asthma: selection by phenotype or stereotype. Eur Respir J 2018; 52: 1801444.

13 Chung KF, Wenzel SE, Brozek JL, et al. International ERS/ATS guidelines on definition, evaluation and treatment of severe asthma. Eur Respir J 2014; 43: 343-373.

14 Flood-Page P, Swenson C, Faiferman I, et al. A study to evaluate safety and efficacy of mepolizumab in patients with moderate persistent asthma. Am J Respir Crit Care Med 2007; 176: 1062-1071.

15 Pavord ID, Haldar P, Bradding P, et al. Mepolizumab in refractory eosinophilic asthma. Thorax 2010; 65: 370

16 Castro M, Corren J, Pavord ID, et al. Dupilumab efficacy and safety in moderate-to-severe uncontrolled asthma. N Engl J Med 2018; 378: 2486-2496.

17 Pavord ID, Chanez P, Criner GJ, et al. Mepolizumab for eosinophilic chronic obstructive pulmonary disease N Engl J Med 2017; 377: 1613-1629.

18 Agusti A, Bel E, Thomas M, et al. Treatable traits: toward precision medicine of chronic airway diseases. Eur Respir J 2016; 47: 410-419.

19 Agusti A, Bafadhel M, Beasley R, et al. Precision medicine in airway diseases: moving to clinical practice. Eur Respir J 2017; 50: 1701655.

20 McDonald VM, Hiles SA, Godbout K, et al. Treatable traits can be identified in a severe asthma registry and predict future exacerbations. Respirology 2018; in press [https://doi.org/10.1111/resp.13389].

21 Baggott C, Beasley R. Triaging treatable traits in asthma. Respirology 2018; in press [https://doi.org/10.1111/resp. 13406].

22 Pavord ID, Beasley R, Agusti A, et al. After asthma: redefining airways diseases. Lancet 2018; 391: 350-400. 